\title{
Rapid, High Throughput, Automated Detection Of SARS-Cov-2 Neutralizing Antibodies Against Native- Like Vaccine And Delta Variant Spike Trimers
}

Narayanaiah Cheedarla

Emory University School of Medicine Hans Verkerke

Emory University School of Medicine

Sindhu Potlapalli

Emory University School of Medicine

Kaleb McLendon

Emory University School of Medicine

Anamika Patel

Emory University School of Medicine

Filipp Frank

Emory University School of Medicine

Gregory Damhorst

Emory University

Huixia Wu

Emory University School of Medicine

William O'Sick

Emory University School of Medicine https://orcid.org/0000-0002-6289-6311

Daniel Graciaa

Emory University

Fuad Hudaib

Emory University School of Medicine

David Alter

Emory University School of Medicine Jeannette Bryksin

Emory University School of Medicine

\section{Eric Ortlund}

Emory University School of Medicine https://orcid.org/0000-0001-8855-3029 Jeannette Guarner

Emory University School of Medicine 
Emory University

\section{Sarita Shah}

Emory University

Wilbur Lam

Georgia Institute of Technology and Emory University

Dawn Mattoon

Quanterix Corporation

Joseph Johnson

Quanterix Corporation https://orcid.org/0000-0001-9728-9792

\section{David Wilson}

Quanterix Corporation

Madhav Dhodapkar

Emory University

\section{Sean Stowell}

Brigham and Women's Hospital

\section{Andrew Neish}

Emory University School of Medicine

John Roback ( $\nabla$ jroback@emory.edu )

Emory University School of Medicine

\section{Article}

Keywords:

Posted Date: February 16th, 2022

DOI: https://doi.org/10.21203/rs.3.rs-1322411/v1

License: (c) (i) This work is licensed under a Creative Commons Attribution 4.0 International License.

Read Full License 
Narayanaiah Cheedarla ${ }^{1,9}$, Hans P. Verkerke ${ }^{1,2,9}$, Sindhu Potlapalli ${ }^{1}$, Kaleb Benjamin McLendon ${ }^{1}$, Anamika

5 Patel $^{3}$, Filipp Frank ${ }^{3}$, Gregory L. Damhorst ${ }^{4}$, Huixia Wu ${ }^{1}$, William Henry O’Sick ${ }^{1}$, Daniel Graciaa ${ }^{4}$, Fuad

6 Hudaib $^{1}$, David N Alter ${ }^{1}$, Jeannette Bryksin ${ }^{1}$, Eric A. Ortlund ${ }^{3}$, Jeanette Guarner ${ }^{1}$, Sara Auld ${ }^{4}$, Sarita Shah $^{4,5}$,

7 Wilbur Lam ${ }^{6}$, Dawn Mattoon ${ }^{7}$, Joseph M Johnson ${ }^{7}$, David H Wilson ${ }^{7}$, Madhav V. Dhodapkar ${ }^{8}$, Sean R.

8 Stowell ${ }^{2 *}$, Andrew S. Neish ${ }^{1 *}$, John D. Roback ${ }^{1^{*}}$.

$9{ }^{1}$ Department of Pathology and Laboratory Medicine, Emory University School of Medicine, Atlanta, GA 1030322 , USA

$11{ }^{2}$ Department of Pathology, Brigham and Women's Hospital, Boston, MA, USA.

$12{ }^{3}$ Department of Biochemistry, Emory University School of Medicine, Atlanta, GA 30322, USA.

$13{ }^{4}$ Department of Medicine, Division of infectious diseases, Emory University, Atlanta, GA 30322, USA.

$14{ }^{5}$ Rollins School of Public Health, Emory University, Atlanta, GA 30322, USA.

$15{ }^{6}$ Wallace H. Coulter Department of Biomedical Engineering, Georgia Institute of Technology and Emory

16 University, Atlanta, GA, USA

$17{ }^{7}$ Quanterix Corporation, 900 Middlesex Turnpike, Billerica, MA 01821

$18{ }^{8}$ Department of Hematology/Medical Oncology, Emory University, Atlanta, GA

$19{ }^{9}$ These authors contributed equally as a first authors

20 *Shared senior authorship, correspondence to srstowell@bwh.harvard.edu, aneish@emory.edu and

21 jroback@emory.edu

23 Abstract

Traditional cellular and live-virus methods for detection of SARS-CoV-2 neutralizing antibodies

25 (nAbs) are labor- and time-intensive, and thus not suited for routine use in the clinical lab to predict vaccine

26 efficacy and natural immune protection. Here, we report the development and validation of a rapid, high

27 throughput method for measuring SARS-CoV-2 nAbs against native-like trimeric spike proteins. This assay

28 uses a blockade of hACE-2 binding (BoAb) approach in an automated digital immunoassay on the

29 Quanterix HD-X platform. BoAb assays using vaccine and delta variant viral strains showed strong

30 correlation with cell-based pseudovirus and live-virus neutralization activity. Importantly, we were able to

31 detect similar patterns of delta variant resistance to neutralization in samples with paired vaccine and delta

32 variant $\mathrm{BoAb}$ measurements. Finally, we screened clinical samples from patients with or without evidence

33 of SARS-CoV-2 exposure by a single-dilution screening version of our assays, finding significant $\mathrm{nAb}$ 
34 activity only in exposed individuals. In principle, these assays offer a rapid, robust, and scalable alternative

35 to time-, skill-, and cost-intensive standard methods for measuring SARS-CoV-2 nAb levels.

Introduction

Levels of neutralizing antibodies (nAbs) against SARS-CoV-2 and other viruses predict vaccine efficacy and immune protection after natural infection ${ }^{1-5}$. In addition, the degree of protection from sterilizing immunity to prevention of severe disease correlates strongly with nAb levels at any given time

41 post vaccination or infection ${ }^{6}$. Thus, the ability to reliably detect and quantify SARS-CoV-2 nAbs at scale

42 is critical in the ongoing public health effort to reach population level protection in the face of waning

43 immunity and a need for boosters ${ }^{7}$. In addition, the emergence of viral variants that escape neutralization

44 by vaccine-induced antibodies underscores the importance of building efficient and reliable pipelines for

$45 \mathrm{nAb}$ assay development as new variants are sequenced and rise to the level of interest or concern (VOI or 46 VOC).

SARS-CoV-2 spike (S) protein is a large homotrimeric glycoprotein, which adopts a metastable

48 prefusion conformation before its high affinity interaction with host-membrane associated angiotensin 49 converting enzyme $2(\text { ACE-2 })^{8-10}$. Native S protein forms two proteolytically cleaved extracellular subunits 50 (S1 and S2), with S1 containing a specific 222 amino acid (AA) receptor binding domain (RBD) that binds 51 to ACE-2 $2^{11-13}$. Thus, S1 promotes receptor recognition and high affinity binding. The S2 subunit, in turn, 52 drives membrane fusion through a fusion peptide (FP), two heptad repeat regions (HR1/2), and a 53 transmembrane domain linked to the cytoplasmic tail ${ }^{14}$. To date, studies of neutralizing antibodies elicited 54 by vaccination and natural infection as well as monoclonal antibody therapies have largely focused on 55 antibodies that bind and inhibit interactions through SARS-CoV-2 RBD ${ }^{15}$. However, studies have also 56 identified targets of neutralizing activity in SARS-CoV-2 S protein outside of the RBD, including regions 57 in S2 proximal to the FP and HR2 ${ }^{16}$. These findings were recently bolstered in a study by Garrett et al. using 58 phage deep mutation scanning (Phage-DMS) to comprehensively interrogate immunodominant epitopes of 59 antibodies in SARS-CoV-2 convalescent plasma as well as routes of antibody escape by the virus. This 
60 study independently identified non-RBD epitopes for neutralizing antibodies in FP and HR2 ${ }^{16}$. Together 61 these findings highlight the importance of closely approximating the native structure and domain 62 organization of spike in any robust assay for SARS-CoV-2 neutralizing antibodies.

63 Current gold-standard assays for measuring nAbs against SARS-CoV-2 require live, replication64 competent wild virus isolates or infectious molecular clones ${ }^{17-18}$. While these assays are important tools for 65 research, they require a biosafety level 3 (BSL3) environment, are difficult to standardize, and are poorly 66 suited for any scaled clinical application due to facilities, personnel, and safety requirements. A second tier 67 of widely accepted nAb assays employ replication incompetent reporter viruses - commonly using 68 backbones derived from either HIV or VSV—-pseudotyped with SARS-CoV-2 Spike (S) ${ }^{19-21}$. These 69 pseudovirus neutralization assays (PNAs) require only BSL2 working conditions and can be scaled for 70 higher throughput. However, both live and pseudoviral assays require use and maintenance of living target 71 cells, which introduces technical variability as well as regulatory complications to clinical testing operations 72 that may seek to employ them. Furthermore, they are manual, labor-intensive assays with turn-around-times 73 of several days. Finally, for lentivirus based PNAs, serum and plasma from patients receiving antiretroviral 74 therapy or pre-exposure prophylaxis for HIV may contain inhibitors of pseudovirus activity non-specific to 75 SARS-CoV-2 $2^{22}$.

76 To address these limitations, we developed and validated a rapid, high throughput, automated 77 blockade of ACE2 binding (BoAb) assay to quantify SARS-CoV-2 nAb activity against both vaccine 78 (Wuhan-1) and delta (B.1.167.2) variant native-like trimeric spike proteins. This assay is performed on the 79 ultrasensitive Quanterix-HDX platform, and is amenable to routine clinical use. We validated our BoAb by 80 comparison to gold standard live virus and pseudovirus neutralization assays as well as clinically in samples

81 from a cohort of SARS-CoV-2 exposed and vaccinated individuals collected during a serosurvey in the

82 spring of 2021. In principle, our approach offers a rapid, adaptable, and scalable solution for detection of 83 nAbs against any SARS-CoV-2 variant, against other viral pathogens, or against emerging viruses of 84 pathogenic potential.

\section{Results}



binding (BoAb). The majority of SARS-CoV-2 neutralizing antibodies prevent viral entry by inhibiting the biochemical interaction between S protein and ACE-2. We therefore designed our assay to detect inhibition of this interaction by nAbs in patient samples or select inhibitors using SARS-CoV-2 spike conjugated beads as targets for binding by a biotinylated ACE-2 detector (Fig. 1A). These reagents were then used in a custom three-step assay on the Quanterix HD-X platform (Fig. 1B). To compare and quantify levels of neutralization from our BoAb assays, we engineered two primary readouts of the assay: an eightpoint titration to identify the $50 \%$ inhibitory dilution or concentration (ID50 or IC50), and a single dilution readout calculated as a percentage of the maximum ACE-2 binding signal for a given spike target bead set. The latter approach was conceived as a potential screening tool for potent neutralizing antibodies while the former titering approach is appropriate for more rigorous comparisons among subjects or between candidate inhibitors (Fig. 1C). and bind with high affinity to ACE-2. In order to present authentic, native-like spike targets for neutralizing antibody detection, we utilized a soluble, stabilized prefusion spike ectodomain construct originally designed in work by Hsieh et $\mathrm{al}^{23}$, but our plasmid DNA sequences are different for WT and 103 Delta strain which were human codon optimized and synthesized at GenScript (Supplementary Text). This 104 construct contains 6 stabilizing proline mutations in S2, which prevent the spontaneous and irreversible 105 formation of a post-fusion state (Fig. 2A). Spike targets for vaccine strain (Wuhan-Hu-1 GenBank: 106 MN908947) and delta variant (B.1.617.2) were produced in human 293F cells and purified by affinity and 107 size exclusion chromatography (SEC). A soluble, human IgG Fc chimera of ACE-2 was produced in a 108 similar system before affinity and SEC. Purity of all in-house generated protein reagents was determined 109 to be $>95 \%$ using reducing SDS-PAGE (Fig. S1). We studied the structures of purified proteins using 110 negative stain electron microscopy NS-EM (Fig 2 B, C) with 2-dimensional class averaging and found that 111 the proteins are formed in trimers. Further, we confirmed that our spike proteins adopted the expected 
112 homotrimeric structures with 3-fold symmetry at the apex and an expected tapering in the S1 to S2 transition

113 (Fig. 2D, E). Finally, we confirmed that our ACE-2 detector bound stably and with high affinity to both of

114 these prefusion constructs using biolayer interferometry (Fig. 2F, G). Both the vaccine strain and delta

115 variant spike reagents bound the ACE2 detector at a similar steady state level and showed stable, slow

116 dissociation rates. Together these data confirm the authentic structure of our spike reagents and their

117 binding activity toward the ACE2 detector used in our BoAb assays.

119 Vaccine strain (Wuh-1) BoAb neutralizing activity correlates strongly with corresponding live virus

120 and pseudovirus neutralization results. To determine the performance of our new test for SARS-CoV-2

121 neutralizing antibodies, we evaluated the correlation between titering results with the vaccine strain BoAb

122 assay versus live virus and pseudovirus neutralization assays using plasma samples from patients vaccinated

123 against COVID-19. Results from our vaccine strain assay showed strong correlation with results from a

124 gold-standard live virus focus reduction neutralization test (FRNT) (Fig. 3A) as well as strong performance

125 in a ROC analysis using the lowest reported $\log$ ID50 (1.17) as a cutoff for activity (AUC 0.94; $\mathrm{P}<0.0001)$

126 (Fig. 3B and 3C). Similarly, our assay correlated strongly with vaccine strain pseudovirus neutralization

127 activity, particularly in samples above the log ID50 limit of quantification (2.0) for our pseudovirus assay

$128\left(\mathrm{R}^{2}=0.72 ; \mathrm{P}<0.001\right)$ (Fig. 3D). Using this pseudovirus LOQ as a cutoff for positivity, we performed a

129 second ROC analysis and comparing vaccine strain BoAb activity in samples below and above the PNA

130 LOQ. Our assay showed robust performance (ROC AUC 0.94; $\mathrm{P}<0.0001$ ) with PNA results as a reference

131 (Fig. 3E and 3F). Our new assay also demonstrated strong correlation with levels of receptor binding 132 domain (RBD) IgG, with samples containing higher levels of neutralizing antibodies as measured by BoAb 133 showing significantly higher levels of RBD binding IgG (Fig. 3G and 3H).

135 Levels of delta variant (B.1.167.2) BoAb neutralization correlate strongly with corresponding live 136 and pseudovirus virus neutralization results and accurately reflect patterns of escape from 137 neutralizing antibodies. We next evaluated the performance of our assay for delta variant (B.1.167.2) 
138 neutralizing activity. We found a strong correlation and robust performance by ROC analysis for our new 139 delta variant BoAb assay ( $\mathrm{R}$ squared of $0.80 ; \mathrm{P}<0.001$ ) as compared to live delta variant neutralizing activity 140 determined by gold standard FRNT assay (Fig. 4A-C). ID50 results from our delta variant assay also 141 correlated strongly with activity in our vaccine strain PNA and the corresponding live virus neutralizing

142 antibody assay though, as expected, with a lower degree of correlation than that seen within strain (R 143 squared of 0.66) (Fig. 4D). ROC analysis revealed a similar performance of the delta variant BoAb assay 144 to the vaccine strain PNA assay using a vaccine strain PNA $\log$ ID50 cutoff of 2 for positivity (Fig. 4E and 145 4F). Delta variant BoAb activity also correlated with vaccine strain RBD binding titers though to a lesser 146 extent (Fig. 4G). Finally, we evaluated the decrement between vaccine strain neutralizing antibody activity 147 and delta variant activity, observed consistently in vaccinated individuals and postulated to be, at least in 148 part, responsible for an increased frequency of delta variant breakthrough infections among vaccinated 149 individuals ${ }^{23}$. Importantly, for each sample we found a similar pattern of decrement in vaccine strain and 150 delta variant BoAb activity compared to live virus vaccine strain and delta variant FRNT results (Fig. 4H 151 and 4I). Together these data suggest that our delta variant BoAb assay correlates strongly with gold 152 standard assays for neutralizing activity and may similarly detect deficits in delta variant specific activity 153 observed among vaccinated individuals and those who experienced infection prior to the emergence of 154 SARS-CoV-2 spike variants with the ability to escape nAbs.

156 Screening for neutralizing antibody activity by single dilution BoAb among SARS-CoV-2 exposed

157 patients. An ideal clinical screening test for SARS-CoV-2 neutralizing activity, in addition to being 158 automated and well correlated with accepted standard assays, should not require limiting-dilution analysis 159 which carries significant costs associated with skilled labor and resources. We therefore generated a single 160 dilution screening test at a sample dilution that was well correlated with live-virus neutralizing activity 161 (1:50) (Fig. 5A). Next, we evaluated the correlation between single-dilution blockade of binding at 1:50 162 with quantitative spike IgG serology, also performed on the Quanterix platform using an EUA serology 163 assay in samples from vaccinated individuals at various times after vaccination. We found a strong linear 
correlation between blockade of binding and levels of spike IgG in samples with spike specific IgG levels

165 between 5 and $100 \mu \mathrm{g} / \mathrm{mL}$. At higher concentrations, blockade of binding was saturated at 100\% inhibition.

166 Significant blockade was not detected in samples with less than $5 \mu \mathrm{g} / \mathrm{mL}$ of spike specific $\operatorname{IgG}$ (Fig. 5B).

167 Finally, the percentage neutralization at a 1:50 dilution was evaluated in a subset of samples from a

168 serosurvey cohort collected in the Emory Healthcare system between January and March of 2021 among

169 inpatients and outpatients who received a blood draw during the relevant encounter. Using available SARS-

170 CoV-2 PCR testing data and serological results, we categorized patients into individuals more likely to have

171 neutralizing activity at the time of sampling (exposed responders) and those unlikely to have nAb activity

172 (unexposed, non-responders). Among 278 patients tested, we identified 115 who were serologically positive

173 with evidence of SARS-CoV-2 exposure. Eighty-five patients were serologically negative without evidence

174 of SARS-CoV-2 exposure at the time of the blood draw. All individuals who screened positive for

175 significant neutralizing activity ( $>50 \%$ inhibition at a 1:50 dilution) in vaccine strain and delta variant single

176 dilutions assays fell into the exposed responder category or had an unknown exposure status at the time of

177 blood draw. Significantly more neutralizing activity was detected against the delta variant in this cohort,

178 perhaps due to the fact that the circulating strain at the time (B1.167-Alpha) $)^{24,25}$ carries many of the same

179 spike mutations as the delta variant (Fig. 5C). Together these data provide proof of concept for use and

180 further validation of our multi-variant BoAb tests as screening tools in patients with evidence of SARS-

181 CoV-2 exposure or vaccination.

\section{Discussion}

We report the development and validation of blockade of binding assays for the detection of nAbs

184 against multiple SARS-CoV-2 variants. Results from our assays correlate strongly with established

185 methods for $\mathrm{nAb}$ detection including live virus FRNT. Unlike these standard methods, our approach is

186 completely automated and rapid, and does not require cell culture, BSL3 facilities, or extensive manual

187 liquid handling. In addition, we employ spike antigens with native trimeric structure in our assays to capture

188 the breadth of epitopes bound by vaccine and infection induced antibody responses. This latter point is 189 particularly important with the roll out of boosters, which purportedly broaden the antibody response. An 
enhancement in neutralizing activity mediated by breadth of epitope specificity would be difficult to detect

191 using subdomain and non-native spike targets.

192 Most viral infections are controlled by functional antibodies, like nAb, that block 193 interactions between viral antigens and host receptors ${ }^{1,24-27}$. In the case of SARS-CoV-2, spike

194 protein interacts with the ACE-2 host cell receptor to enter host cells. Before interacting with host 195 receptor, the spike antigen exists in a prefusion state. Conformational changes occur in the spike 196 protein once it binds to ACE-2 receptor. Based on this finding, most assays were developed to 197 detect nAbs in blood samples by incubating plasma with pseudo-typed viruses or live viruses prior 198 to co-culture with target cells ${ }^{28,29}$. It is well understood that viruses maintain the prefusion state of 199 viral antigens (spike for SARS-CoV-2 and Env for HIV-1 and HIV-2) before interacting with host 200 receptor, and most nAbs target the prefusion state of viral antigen ${ }^{30-32}$. Based on this model, we 201 established an assay for the identification of nAbs against the prefusion state of SARS-CoV-2 202 spike protein that block interactions with human ACE-2 receptor. We have observed that the 203 purified version of spike protein from Wuh-1 (WT) and Delta used in these studies maintained 204 structures with one RBD up (opened confirmation) and two RBD down (closed confirmation) 205 which was previously observed by Z Ke et al., when they studied spike structures on intact virion 206 by cryo-EM images ${ }^{9}$. Thus, the purified spike proteins used in this assay maintain the prerequisite 207 conformational state for the RBD-ACE2 interaction. Further, we observed that the spike proteins 208 showed strong hACE-2 interaction by BLI.

209 For the initial analysis of BoAb assay, we used samples from Nooka et al., ${ }^{33}$ who studied 210 the neutralization determinants in myeloma patients. With this cohort, we observed that results in 211 the BoAb assay strongly correlated with both live virus neutralization (Wuhan $\mathrm{R}^{2}=0.82$ and $212 \mathrm{p}=<0.001$; Delta $\mathrm{R}^{2}=0.80$ and $\mathrm{p}=<0.001$ ) and pseudovirus neutralization results (Wuhan $\mathrm{R}^{2}=$ 2130.72 and $\mathrm{p}=<0.001$; Delta $\mathrm{R}^{2}=0.66$ and $\left.\mathrm{p}=<0.001\right)$. Interestingly, we also identified significant 
214 correlation of BoAb results with RBD endpoint titers for both Wuhan $(\mathrm{p}=<0.001)$ and Delta strains

$215(\mathrm{p}=0.002)$. These results agree with other studies in which RBD titers are strongly correlated with

216 neutralization titers ${ }^{34-36}$. In addition, we determined that the BoAb assay showed sensitivity $>94 \%$

217 against the Wuhan strain and $>90 \%$ against Delta strain, both with $100 \%$ specificity. In general,

218 neutralization against Delta strain was lower than for Wuhan strain, similarly to results from the

219 BoAb assay.

220 We evaluated the utility of our BoAb assay in a second cohort of samples from a serological

221 survey of patients requiring a blood draw in the Emory Healthcare system from January to March

222 of 2021. We used available COVID-19 PCR testing results and combined serological status to

223 categorize patients who were likely to have been exposed to SARS-CoV-2 and to have developed

224 a humoral immune response against the virus. We hypothesized that this group of exposed

225 responders had the highest pre-test probability in the cohort of harboring SARS-CoV-2

226 neutralizing activity and screened them using a 1:50 single dilution version of our BoAb assay. In

227 agreement with our hypothesis, we observed strong neutralization against SARS-CoV-2 spike that

228 was consistent with the patients' exposure status. Therefore, our data is strongly aligned with

229 correlations of antibody responses vs neutralization responses against SARS-CoV-2 ${ }^{37-39}$. The

230 rapid, automated, high-throughput BoAb assay described here may be useful for large-scale

231 quantification of nAbs against SARS-CoV-2 variants for both clinical and investigational uses.

232 It should be noted that our study was limited by availability of gold-standard live-virus neutralizing

233 antibody data and a need to directly correlate activity measured in our assay with known correlates of nAb

234 activity in gold standard cell-based assays. While our data suggest that biochemical neutralization as

235 measured by BoAb correlates well with results from these more established tests, additional work is needed

236 to evaluate the implications of this association for vaccine efficacy and protection after natural infection. 
240 Samples. Samples were sourced from various studies with IRB 00001663 at Emory University after

241 obtaining the approval and consent from Institutional Review Board and samples $(\mathrm{n}=300)$ are tested on The

242 Quanterix HD-X which uses single molecule arrays (SIMOAS) of femtoliter-sized reaction chambers

243 etched into a disk to detect single enzyme labeled proteins. Our assay uses a 3-step ELISA that begins with

244 an initial incubation of the sample and spike-conjugated magnetic bead reagent. Then the beads are

245 separated magnetically and washed. Following a biotinylated ACE2 detector is added and incubated. A

246 second magnetic separation and wash are performed. Followed by a streptavidin beta-galactosidase

247 incubation and third wash. A resorufin B-D-galactopyranoside substrate is added and the reaction chamber

248 is sealed with oil. Any spike antigen that was able to attract a detector antibody will lead to the formation

249 of an SBG/RGP fluorescent product that is counted digitally. The instrument can handle up to 288 replicates

250 at a time.

251 Pseudovirus neutralization assay (PNA). Neutralization activities against SARS-CoV-2 WT (Wuh-1) and

252 Delta (B.1.617.2) strains were measured in a single-round-of-infection assay with pseudo viruses, as

253 previously described ${ }^{33}$. Briefly, to produce SARS-CoV-2 WT and Delta pseudoviruses, an expression

254 plasmid bearing codon optimized SARS-CoV-2 full-length S plasmid (parental sequence Wuhan-1,

255 Genbank \#: MN908947.3) was co-transfected into HEK293T cells (ATCC\#CRL-11268) with plasmids

256 encoding non-surface proteins for lentivirus production and a lentiviral backbone plasmid expressing a

257 Luciferase-IRES-ZsGreen reporter, HIV-1 Tat and Rev packing plasmids (BEI Resources) and

258 pseudoviruses harvested after 48 hours of post transfection and performed titration. Pseudoviruses were

259 mixed with serial dilutions of plasma or antibodies and then added to monolayers of ACE-2-overexpressing

260 293T cells (BEI Resources), in duplicate. 24 hours after infection, cells were lysed, luciferase was activated

261 with the Luciferase Assay System (Promega), and relative light units (RLU) were measured on a synergy

262 Biotek reader. 
264 Protein expression and purification. Trimeric SARS-CoV-2 Spike (Wuh-1 and Delta B.1.617.2) as well

265 as Angiotensin Converting Enzyme-2 (ACE-2)-IgFC chimera proteins were produced by transfection in 266 FreeStyle 293-F cells using plasmids (MN908947 for Wuhan-1 and Delta strains, and NM_021804.3 for 267 hACE-2). Briefly, FreeStyle 293F cells were seeded at a density of 2E6 cells/ml in Expi293 expression 268 media and incubated with shaking on at $37^{\circ} \mathrm{C}$ and $127 \mathrm{rpm}$ with $8 \% \mathrm{CO} 2$ overnight. The following day, $2692.5 \mathrm{E} 6$ cells $/ \mathrm{ml}$ were transfected using ExpiFectamine ${ }^{\mathrm{TM}} 293$ transfection reagent (ThermoFisher, cat. no. 270 A14524) according to the manufacturer protocol. Transfected cells were then incubated with orbital shaking 271 for $4-5$ days at $37{ }^{\circ} \mathrm{C}, 127 \mathrm{rpm}, 8 \% \mathrm{CO} 2$. Supernatants containing secreted trimeric ectodomains were 272 collected by centrifugation at $4,000 \mathrm{xg}$ for 20 minutes at $4^{\circ} \mathrm{C}$. Clarified supernatants were then filtered using 273 a $0.22 \mu \mathrm{m}$ stericup filter (ThermoFisher, cat.no. 290-4520) and loaded onto pre-equilibrated affinity 274 columns for protein purification. The SARS-CoV-2 Spike trimer and ACE-2 proteins were purified using 275 His-Pur Ni-NTA resin (ThermoFisher, cat.no. 88221) and Protein-G Agarose (ThermoFisher, cat.no. 276 20399) respectively. Briefly, His-Pur Ni-NTA resin was washed twice with PBS by centrifugation at $2772000 x g$ for $10 \mathrm{~min}$. The resin was resuspended with the spike-trimer supernatant and incubated for 2 hours 278 on a shaker at RT. Gravity flow columns were then loaded with supernatant-resin mixture and washed 279 (25mM Imidazole, $6.7 \mathrm{mM} \mathrm{NaH}_{2} \mathrm{PO}_{4} \cdot \mathrm{H}_{2} \mathrm{O}$ and $300 \mathrm{mM} \mathrm{NaCl}$ in PBS) four times, after which the protein 280 was eluted in elution buffer (235 mM Imidazole, $6.7 \mathrm{mM} \mathrm{NaH}_{2} \mathrm{PO}_{4} \cdot \mathrm{H}_{2} \mathrm{O}$ and $300 \mathrm{mM} \mathrm{NaCl}$ in PBS). Eluted 281 protein was dialyzed against PBS using Slide-A-lyzer Dialysis Cassette (ThermoScientific, Cat\# 66030) 282 and concentrated using $100 \mathrm{kDa}$ Amicon Centrifugal Filter Unit, at $2000 \mathrm{~g}$ at $4^{\circ} \mathrm{C}$. The concentrated protein 283 eluate was then run and fractionated on a Sepharose 600 (GE Healthcare) column on an Akta ${ }^{\mathrm{TM}}$ Pure (GE 284 Healthcare). Fractions corresponding to the molecular weight of each protein were pooled and concentrated 285 as described above. Proteins were quantified by BCA Protein Assay Kit (Pierce) and quality was confirmed 286 by SDS-PAGE (supplementary Figure 1).

288 ACE-2 protein expression and purification. The soluble ACE-2 IgFC chimera was expressed as 289 described above. Clarified supernatants were diluted 1:1 with binding buffer before loading on a protein $\mathrm{g}$ 
gravity flow column, pre-equilibrated with $10 \mathrm{ml}$ of binding buffer (Pierce cat.no.21011). Columns were

291 washed with $20 \mathrm{ml}$ of binding buffer, and the protein was eluted in $40 \mathrm{ml}$ of elution buffer. Following 292 elution, samples were first neutralized to $\mathrm{pH} 7.5$ using $1 \mathrm{M}$ Tris, pH 9.0. Eluted protein was dialyzed against 293 50mM Tris (pH7.5), 150mM NaCl using a Slide-A-lyzer Dialysis Cassette (ThermoScientific, Cat\# 66030)

294 and concentrated using $50 \mathrm{kDa}$ Amicon Centrifugal Filter Unit, at $2000 \mathrm{~g}$ at $4^{\circ} \mathrm{C}$. Size exclusion 295 chromatography and quality control was performed on the concentrated protein as described above.

Assessment of Spike-ACE-2 binding by biolayer interferometry. 6x His-tagged spike was diluted to 50 $\mu \mathrm{g} / \mathrm{mL}$ in PBS before immobilization on nickel NTA biosensors (fortebio). Association of ACE-2 was monitored using and OctetRED96e instrument (fortebio) in 2-fold dilutions series starting at $100 \mu \mathrm{g} / \mathrm{mL}$ for 600 s followed by dissociation in PBS for 500s. Tips were regenerated using $10 \mathrm{mM}$ glycine and regenerated in $10 \mathrm{mM} \mathrm{NiCl} 2$ before re-loading with equivalent concentrations of spike.

Generation of detector and conjugated beads. ACE-2 detector biotinylation and spike bead conjugation were performed per the Quanterix Homebrew Detection Antibody Biotinylation and Bead Conjugation Protocols. prior to mixing at $1 \mathrm{mg} / \mathrm{mL}$ with a $40 \mathrm{x}$ challenge ratio of NHS-PEG4-biotin for 30 minutes at room

310 temperature. Cleanup of the biotinylated detection reagent was achieved by a further round of amicon 311 filtration following recovery in biotinylation reaction buffer and determination of protein concentration. A 312 final detector concentration of $0.5 \mu \mathrm{g} / \mathrm{mL}$ was used in the assay.

314 Paramagenetic beads were activated after washing with bead conjugation buffer using 9ug EDC (10mg/mL)

315 in a final bead volume of $300 \mu \mathrm{l}$ containing $4.2 \mathrm{E} 8$ beads for 30 minutes at $4{ }^{\circ} \mathrm{C}$ with rocking. Following 
316 activation, beads were washed with Bead Conjugation Buffer and $300 \mu \mathrm{l}$ cold spike at $0.2 \mathrm{mg} / \mathrm{mL}$ to the

317 beads followed by incubation at $4{ }^{\circ} \mathrm{C}$ with rocking for 2 hours. Beads were then washed and blocked for 45

318 minutes at room temperature, followed by a final wash and resuspension in $300 \mu 1$ bead diluent. Spike

319 capture beads were stored at $4{ }^{\circ} \mathrm{C}$ until use in the assay.

321 Negative Stain sample preparation, data collection and data analysis. Spike protein was diluted to $3220.05 \mathrm{mg} / \mathrm{ml}$ in PBS prior to grid preparation. A $3 \mu \mathrm{L}$ drop of diluted protein applied to previously glow323 discharged, carbon coated grids for $\sim 60 \mathrm{sec}$, blotted and washed twice with water, stained with $0.75 \%$ 324 uranyl formate, blotted and air dried. Between 25-35 images were collected on Talos L120C microscope 325 (Thermo Fisher, Waltham, MA) at 73,000 magnification and $1.97 \AA$ pixel size. Relion-3.1 was used for 326 particle picking, 2D classification and 3D reconstruction [PMID: 23000701].

328 Statistical Analysis. GraphPad PRISM version 9 was used to perform the statistical analysis. Correlation 329 between the assays was performed by Pearson $r$ correlation method and linear regression analysis. All 330 statistical tests were two-sided, unless otherwise noted, and statistical significance was assessed at the $331 * \mathrm{P}<0.05, * * \mathrm{P}<0.01, * * * \mathrm{P}<0.001, * * * * \mathrm{P}<0.0001$ and further details are provided in the figure legend where 332 analysis was performed.

\section{Acknowledgments}

336 We would like to thank Cato, Lee from Eric Ortlund lab for help in processing SEC for protein purification 337 and we also acknowledge Amit Joshi, Todd Glynn and Danielle Svancara from Quanterix team for their 338 tremendous support while developing assay on HDX instrument. We acknowledge BEI Resources for 339 providing plasmid (NR-52309) and 293T-ACE-2 cell line (NR-52511). The Robert P. Apkarian Integrated

340 Electron Microscopy Core (IEMC) at Emory University is subsidized by the School of Medicine and Emory 341 College of Arts and Sciences. Additional support is provided by the Georgia Clinical \& Translational 
342 Science Alliance of the National Institutes of Health under award number UL1TR000454. We would like

343 to acknowledge Connie Arthur and Cheryl Meier for their fabulous support with serosurvey specimen 344 processing.

347 Authors Contributions

349 N.C., H.V., A.S.N., and J.D.R. contributed to the acquisition, analysis, and interpretation of the data, S.P., 350 K.B.M., W.H.Os., and F.H. interpretation of data, A.P., and F.F. involved in the interpretation of the NS351 EM images, G.D., H.W., J.B., J.G., S.A., S.S., and M.V.D provided samples from Emory Healthcare, 352 D.N.A., E.O., W.L., S.R.S., A.S.N and J.D.R. contributed to the data analysis, and A.S.N., and J.D.R 353 received grants from NIH/NCI, 1 U54 CA260563-01: Immune regulation of COVID-19 infection in cancer 354 and autoimmunity and Emory CURE grant from Emory University, Atlanta, USA.

Declaration of Interests

357 N.C., H.V., S.P., K.B.M., W.H.Os., H.W., A.S.N., and J.D.R. are co-inventors of BoAb assay technology. 358 Emory University filed a patent on this technology.

\section{Reference:}

361 1. Tea F, Ospina Stella A, Aggarwal A, Ross Darley D, Pilli D, Vitale D, et al. (2021) SARS-CoV-2 362 neutralizing antibodies: Longevity, breadth, and evasion by emerging viral variants. PLoS Med 363 18(7): e1003656. https://doi.org/10.1371/journal.pmed.1003656

364 2. Khoury DS, Cromer D, Reynaldi A, Schlub TE, Wheatley AK, Juno JA, Subbarao K, Kent SJ, 365 Triccas JA, Davenport MP. Neutralizing antibody levels are highly predictive of immune protection 366 from symptomatic SARS-CoV-2 infection. Nat Med. 2021 Jul;27(7):1205-1211. doi: 10.1038/s41591-021-01377-8. Epub 2021 May 17. PMID: 34002089. 
3. Overbaugh, J., \& Morris, L. (2012). The Antibody Response against HIV-1. Cold Spring Harbor perspectives in medicine, 2(1), a007039. https://doi.org/10.1101/cshperspect.a007039.

4. Tan C-W, Chia W-N, Young BE, et al. Pan-Sarbecovirus Neutralizing Antibodies in BNT162b2Immunized SARS-CoV-1 Survivors. New England Journal of Medicine 2021; Available at: https://doi.org/10.1056/NEJMoa2108453. Accessed 26 September 2021.

5. Wang, Z., Muecksch, F., Schaefer-Babajew, D. et al. Naturally enhanced neutralizing breadth against SARS-CoV-2 one year after infection. Nature 595, 426-431 (2021). https://doi.org/10.1038/s41586-021-03696-9 )

6. Milne G, Hames T, Scotton C, Gent N, Johnsen A, Anderson RM, Ward T. Does infection with or vaccination against SARS-CoV-2 lead to lasting immunity? Lancet Respir Med. 2021 Oct 21:S2213-2600(21)00407-0. doi: 10.1016/S2213-2600(21)00407-0. Epub ahead of print. PMID: 34688434; PMCID: PMC8530467.

7. Levine-Tiefenbrun, M., Yelin, I., Alapi, H. et al. Viral loads of Delta-variant SARS-CoV-2 breakthrough infections after vaccination and booster with BNT162b2. Nat Med (2021). https://doi.org/10.1038/s41591-021-01575-4.

8. Wang MY, Zhao R, Gao LJ, Gao XF, Wang DP, Cao JM. SARS-CoV-2: Structure, Biology, and Structure-Based Therapeutics Development. Front Cell Infect Microbiol. 2020 Nov 25;10:587269. doi: 10.3389/fcimb.2020.587269. PMID: 33324574; PMCID: PMC7723891.

9. Ke Z, Oton J, Qu K, Cortese M, Zila V, McKeane L, Nakane T, Zivanov J, Neufeldt CJ, Cerikan B, Lu JM, Peukes J, Xiong X, Kräusslich HG, Scheres SHW, Bartenschlager R, Briggs JAG. Structures and distributions of SARS-CoV-2 spike proteins on intact virions. Nature. 2020 Dec;588(7838):498-502. doi: 10.1038/s41586-020-2665-2. Epub 2020 Aug 17. PMID: 32805734; PMCID: PMC7116492.

10. Yao H, Song Y, Chen Y, Wu N, Xu J, Sun C, Zhang J, Weng T, Zhang Z, Wu Z, Cheng L, Shi D, Lu X, Lei J, Crispin M, Shi Y, Li L, Li S. Molecular Architecture of the SARS-CoV-2 Virus. Cell. 
2020 Oct 29;183(3):730-738.e13. doi: 10.1016/j.cell.2020.09.018. Epub 2020 Sep 6. PMID: 32979942; PMCID: PMC7474903.

11. Seyran M, Takayama K, Uversky VN, Lundstrom K, Palù G, Sherchan SP, Attrish D, Rezaei N, Aljabali AAA, Ghosh S, Pizzol D, Chauhan G, Adadi P, Mohamed Abd El-Aziz T, Soares AG, Kandimalla R, Tambuwala M, Hassan SS, Azad GK, Pal Choudhury P, Baetas-da-Cruz W, Serrano-Aroca Á, Brufsky AM, Uhal BD. The structural basis of accelerated host cell entry by SARS-CoV-2†. FEBS J. 2021 Sep;288(17):5010-5020. doi: 10.1111/febs.15651. Epub 2020 Dec 14. PMID: 33264497; PMCID: PMC7753708.

12. Tomassetti F, Nuccetelli M, Sarubbi S, Gisone F, Ciotti M, Spinazzola F, Ricotta C, Cagnoli M, Borgatti M, Iannetta M, Andreoni M, Calugi G, Pieri M, Bernardini S. Evaluation of S-RBD and

13. Lan J, Ge J, Yu J, Shan S, Zhou H, Fan S, Zhang Q, Shi X, Wang Q, Zhang L, Wang X. Structure of the SARS-CoV-2 spike receptor-binding domain bound to the ACE2 receptor. Nature. 2020 May;581(7807):215-220. doi: 10.1038/s41586-020-2180-5. Epub 2020 Mar 30. PMID: 32225176. high specificity ACE-2-binding antibodies on SARS-CoV-2 patients after six months from infection. Int Immunopharmacol. 2021 Oct;99:108013. doi: 10.1016/j.intimp.2021.108013. Epub 2021 Jul 27. PMID: 34339963; PMCID: PMC8313542.

14. Xia X. Domains and Functions of Spike Protein in Sars-Cov-2 in the Context of Vaccine Design. Viruses. 2021 Jan 14;13(1):109. doi: 10.3390/v13010109. PMID: 33466921; PMCID: PMC7829931.

15. Piccoli L, Park YJ, Tortorici MA, Czudnochowski N, Walls AC, Beltramello M, Silacci-Fregni C, Pinto D, Rosen LE, Bowen JE, Acton OJ, Jaconi S, Guarino B, Minola A, Zatta F, Sprugasci N, Bassi J, Peter A, De Marco A, Nix JC, Mele F, Jovic S, Rodriguez BF, Gupta SV, Jin F, Piumatti G, Lo Presti G, Pellanda AF, Biggiogero M, Tarkowski M, Pizzuto MS, Cameroni E, HavenarDaughton C, Smithey M, Hong D, Lepori V, Albanese E, Ceschi A, Bernasconi E, Elzi L, Ferrari P, Garzoni C, Riva A, Snell G, Sallusto F, Fink K, Virgin HW, Lanzavecchia A, Corti D, Veesler D. Mapping Neutralizing and Immunodominant Sites on the SARS-CoV-2 Spike Receptor-Binding 
Domain by Structure-Guided High-Resolution Serology. Cell. 2020 Nov 12;183(4):10241042.e21. doi: 10.1016/j.cell.2020.09.037. Epub 2020 Sep 16. PMID: 32991844; PMCID: PMC7494283.

16. Garrett ME, Galloway J, Chu HY, Itell HL, Stoddard CI, Wolf CR, Logue JK, McDonald D, Matsen FA 4th, Overbaugh J. High resolution profiling of pathways of escape for SARS-CoV-2 spikebinding antibodies. bioRxiv [Preprint]. 2020 Nov 16:2020.11.16.385278. doi: 10.1101/2020.11.16.385278. Update in: Cell. 2021 May 4;: PMID: 33236010; PMCID: PMC7685320.

17. Bewley, K.R., Coombes, N.S., Gagnon, L. et al. Quantification of SARS-CoV-2 neutralizing antibody by wild-type plaque reduction neutralization, microneutralization and pseudotyped virus neutralization assays. Nat Protoc 16, 3114-3140 (2021). https://doi.org/10.1038/s41596-021$\underline{00536-y}$

18. Suthar MS, Zimmerman MG, Kauffman RC, Mantus G, Linderman SL, Hudson WH, Vanderheiden A, Nyhoff L, Davis CW, Adekunle O, Affer M, Sherman M, Reynolds S, Verkerke HP, Alter DN, Guarner J, Bryksin J, Horwath MC, Arthur CM, Saakadze N, Smith GH, Edupuganti S, Scherer EM, Hellmeister K, Cheng A, Morales JA, Neish AS, Stowell SR, Frank F, Ortlund E, Anderson EJ, Menachery VD, Rouphael N, Mehta AK, Stephens DS, Ahmed R, Roback JD, Wrammert J. Rapid Generation of Neutralizing Antibody Responses in COVID-19 Patients. Cell Rep Med. 2020 Jun 23;1(3):100040. doi: 10.1016/j.xcrm.2020.100040. Epub 2020 Jun 8. PMID: 32835303; PMCID: PMC7276302.

19. Xiong HL, Wu YT, Cao JL, Yang R, Liu YX, Ma J, Qiao XY, Yao XY, Zhang BH, Zhang YL, Hou WH, Shi Y, Xu JJ, Zhang L, Wang SJ, Fu BR, Yang T, Ge SX, Zhang J, Yuan Q, Huang BY, Li ZY, Zhang TY, Xia NS. Robust neutralization assay based on SARS-CoV-2 S-protein-bearing vesicular stomatitis virus (VSV) pseudovirus and ACE2-overexpressing BHK21 cells. Emerg Microbes Infect. 2020 Dec;9(1):2105-2113. doi: 10.1080/22221751.2020.1815589. PMID: 32893735; PMCID: PMC7534347. 
20. Nie J, Li Q, Wu J, Zhao C, Hao H, Liu H, Zhang L, Nie L, Qin H, Wang M, Lu Q, Li X, Sun Q, Liu J, Fan C, Huang W, Xu M, Wang Y. Establishment and validation of a pseudovirus neutralization assay for SARS-CoV-2. Emerg Microbes Infect. 2020 Dec;9(1):680-686. doi: 10.1080/22221751.2020.1743767. PMID: 32207377; PMCID: PMC7144318.

21. Hu J, Gao Q, He C, Huang A, Tang N, Wang K. Development of cell-based pseudovirus entry assay to identify potential viral entry inhibitors and neutralizing antibodies against SARS-CoV-2. Genes Dis. 2020 Dec;7(4):551-557. doi: 10.1016/j.gendis.2020.07.006. Epub 2020 Jul 17. PMID: 32837985; PMCID: PMC7366953.

22. Cheresiz SV, Grigoryev IV, Semenova EA, Pustylnyak VO, Vlasov VV, Pokrovsky AG. A pseudovirus system for the testing of antiviral activity of compounds in different cell lines. Dokl Biochem Biophys. 2010 Nov-Dec;435:295-8. doi: 10.1134/S1607672910060049. Epub 2010 Dec 24. PMID: 21184297.

23. Hsieh CL, Goldsmith JA, Schaub JM, DiVenere AM, Kuo HC, Javanmardi K, Le KC, Wrapp D, Lee AG, Liu Y, Chou CW, Byrne PO, Hjorth CK, Johnson NV, Ludes-Meyers J, Nguyen AW, Park J, Wang N, Amengor D, Lavinder JJ, Ippolito GC, Maynard JA, Finkelstein IJ, McLellan JS. Structure-based design of prefusion-stabilized SARS-CoV-2 spikes. Science. 2020 Sep 18;369(6510):1501-1505. doi: 10.1126/science.abd0826. Epub 2020 Jul 23. PMID: 32703906; PMCID: PMC7402631.

24. Cheedarla, N.; Hanna, L.E. Chapter 7-Functional and Protective Role of Neutralizing Antibodies (NAbs) Against Viral Infections. In Recent Developments in Applied Microbiology and Biochemistry; Buddolla, V., Ed.; Academic Press: Cambridge, MA, USA, 2019; pp. 83-93.

25. Murin CD, Wilson IA, Ward AB. Antibody responses to viral infections: a structural perspective across three different enveloped viruses. Nat Microbiol. 2019 May;4(5):734-747. doi: 10.1038/s41564-019-0392-y. Epub 2019 Mar 18. PMID: 30886356; PMCID: PMC6818971.

26. Addetia A, Crawford KHD, Dingens A, Zhu H, Roychoudhury P, Huang ML, Jerome KR, Bloom JD, Greninger AL. Neutralizing Antibodies Correlate with Protection from SARS-CoV-2 in 

21;58(11):e02107-20. doi: 10.1128/JCM.02107-20. PMID: 32826322; PMCID: PMC7587101. 27. Padilla-Quirarte HO, Lopez-Guerrero DV, Gutierrez-Xicotencatl L, Esquivel-Guadarrama F. Protective Antibodies Against Influenza Proteins. Front Immunol. 2019 Jul 18;10:1677. doi: 10.3389/fimmu.2019.01677. PMID: 31379866; PMCID: PMC6657620.

28. Nie J, Li Q, Wu J, Zhao C, Hao H, Liu H, Zhang L, Nie L, Qin H, Wang M, Lu Q, Li X, Sun Q, Liu J, Fan C, Huang W, Xu M, Wang Y. Establishment and validation of a pseudovirus neutralization assay for SARS-CoV-2. Emerg Microbes Infect. 2020 Dec;9(1):680-686. doi: 10.1080/22221751.2020.1743767. PMID: 32207377; PMCID: PMC7144318.

29. Montefiori DC, Roederer M, Morris L, Seaman MS. Neutralization tiers of HIV-1. Curr Opin HIV

30. Lan J, Ge J, Yu J, Shan S, Zhou H, Fan S, Zhang Q, Shi X, Wang Q, Zhang L, Wang X. Structure of the SARS-CoV-2 spike receptor-binding domain bound to the ACE2 receptor. Nature. 2020 May;581(7807):215-220. doi: 10.1038/s41586-020-2180-5. Epub 2020 Mar 30. PMID: 32225176.

31. Pancera M, Zhou T, Druz A, Georgiev IS, Soto C, Gorman J, Huang J, Acharya P, Chuang GY, Ofek G, Stewart-Jones GB, Stuckey J, Bailer RT, Joyce MG, Louder MK, Tumba N, Yang Y, Zhang B, Cohen MS, Haynes BF, Mascola JR, Morris L, Munro JB, Blanchard SC, Mothes W, Connors M, Kwong PD. Structure and immune recognition of trimeric pre-fusion HIV-1 Env. Nature. 2014 Oct 23;514(7523):455-61. doi: 10.1038/nature13808. Epub 2014 Oct 8. PMID: 25296255; PMCID: PMC4348022.

32. Rutten L, Lai YT, Blokland S, Truan D, Bisschop IJM, Strokappe NM, Koornneef A, van Manen D, Chuang GY, Farney SK, Schuitemaker H, Kwong PD, Langedijk JPM. A Universal Approach to Optimize the Folding and Stability of Prefusion-Closed HIV-1 Envelope Trimers. Cell Rep. 2018 Apr 10;23(2):584-595. doi: 10.1016/j.celrep.2018.03.061. PMID: 29642014; PMCID: PMC6010203. 
33. Nooka et al., Determinants Of Neutralizing Antibody Response Following SARS CoV-2 Vaccination In Myeloma (Manuscript accepted to publish in Journal of Clinical Oncology)

34. Ripperger TJ, Uhrlaub JL, Watanabe M, Wong R, Castaneda Y, Pizzato HA, Thompson MR, Bradshaw C, Weinkauf CC, Bime C, Erickson HL, Knox K, Bixby B, Parthasarathy S, Chaudhary S, Natt B, Cristan E, El Aini T, Rischard F, Campion J, Chopra M, Insel M, Sam A, Knepler JL, Capaldi AP, Spier CM, Dake MD, Edwards T, Kaplan ME, Scott SJ, Hypes C, Mosier J, Harris DT, LaFleur BJ, Sprissler R, Nikolich-Žugich J, Bhattacharya D. Orthogonal SARS-CoV-2 Serological Assays Enable Surveillance of Low-Prevalence Communities and Reveal Durable Humoral Immunity. Immunity. 2020 Nov 17;53(5):925-933.e4. doi: 10.1016/j.immuni.2020.10.004. Epub 2020 Oct 14. PMID: 33129373; PMCID: PMC7554472.

35. Klein SL, Pekosz A, Park HS, Ursin RL, Shapiro JR, Benner SE, Littlefield K, Kumar S, Naik HM, Betenbaugh MJ, Shrestha R, Wu AA, Hughes RM, Burgess I, Caturegli P, Laeyendecker O, Quinn TC, Sullivan D, Shoham S, Redd AD, Bloch EM, Casadevall A, Tobian AA. Sex, age, and hospitalization drive antibody responses in a COVID-19 convalescent plasma donor population. J Clin Invest. 2020 Nov 2;130(11):6141-6150. doi: 10.1172/JCI142004. PMID: 32764200; PMCID: PMC7598041.

36. Liu L, Wang P, Nair MS, Yu J, Rapp M, Wang Q, Luo Y, Chan JF, Sahi V, Figueroa A, Guo XV, Cerutti G, Bimela J, Gorman J, Zhou T, Chen Z, Yuen KY, Kwong PD, Sodroski JG, Yin MT, Sheng Z, Huang Y, Shapiro L, Ho DD. Potent neutralizing antibodies against multiple epitopes on SARS-CoV-2 spike. Nature. 2020 Aug;584(7821):450-456. doi: 10.1038/s41586-020-2571-7. Epub 2020 Jul 22. PMID: 32698192.

37. Gaebler C, Wang Z, Lorenzi JCC, Muecksch F, Finkin S, Tokuyama M, Cho A, Jankovic M, Schaefer-Babajew D, Oliveira TY, Cipolla M, Viant C, Barnes CO, Bram Y, Breton G, Hägglöf T, Mendoza P, Hurley A, Turroja M, Gordon K, Millard KG, Ramos V, Schmidt F, Weisblum Y, Jha D, Tankelevich M, Martinez-Delgado G, Yee J, Patel R, Dizon J, Unson-O'Brien C, Shimeliovich I, Robbiani DF, Zhao Z, Gazumyan A, Schwartz RE, Hatziioannou T, Bjorkman PJ, Mehandru S, 
Bieniasz PD, Caskey M, Nussenzweig MC. Evolution of antibody immunity to SARS-CoV-2. Nature. 2021 Mar;591(7851):639-644. doi: 10.1038/s41586-021-03207-w. Epub 2021 Jan 18. PMID: 33461210; PMCID: PMC8221082.

38. Robbiani DF, Gaebler C, Muecksch F, Lorenzi JCC, Wang Z, Cho A, Agudelo M, Barnes CO, Gazumyan A, Finkin S, Hägglöf T, Oliveira TY, Viant C, Hurley A, Hoffmann HH, Millard KG, Kost RG, Cipolla M, Gordon K, Bianchini F, Chen ST, Ramos V, Patel R, Dizon J, Shimeliovich I, Mendoza P, Hartweger H, Nogueira L, Pack M, Horowitz J, Schmidt F, Weisblum Y, Michailidis E, Ashbrook AW, Waltari E, Pak JE, Huey-Tubman KE, Koranda N, Hoffman PR, West AP Jr, Rice CM, Hatziioannou T, Bjorkman PJ, Bieniasz PD, Caskey M, Nussenzweig MC. Convergent antibody responses to SARS-CoV-2 in convalescent individuals. Nature. 2020 Aug;584(7821):437-442. doi: 10.1038/s41586-020-2456-9. Epub 2020 Jun 18. PMID: 32555388; PMCID: PMC7442695.

39. Liu Z, Xu W, Xia S, Gu C, Wang X, Wang Q, Zhou J, Wu Y, Cai X, Qu D, Ying T, Xie Y, Lu L, Yuan Z, Jiang S. RBD-Fc-based COVID-19 vaccine candidate induces highly potent SARS-CoV2 neutralizing antibody response. Signal Transduct Target Ther. 2020 Nov 27;5(1):282. doi: 


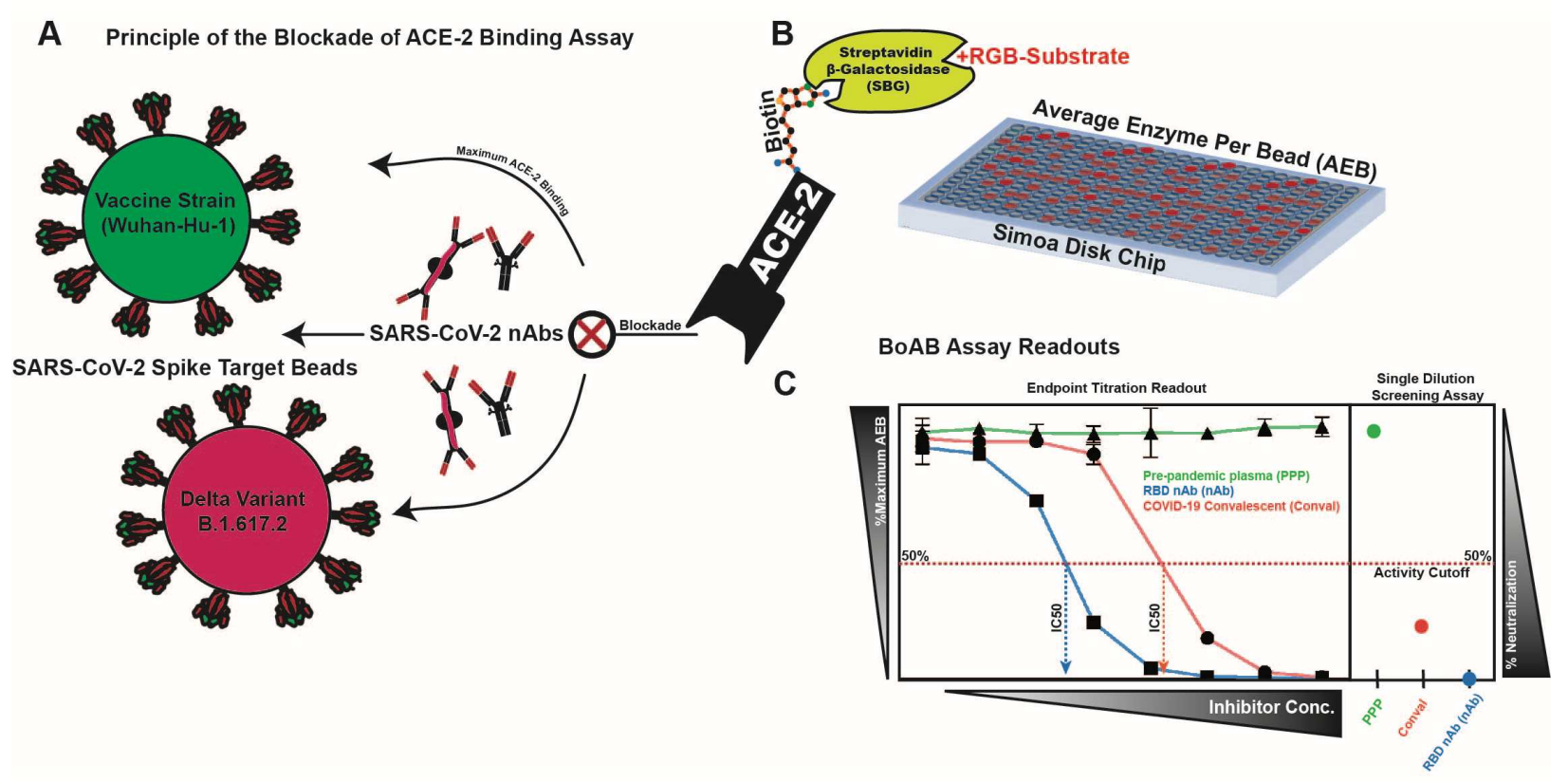

Figure 1. Blockade of ACE-2 Binding (BoAb) assay design. A schematic of our blockade of binding assay for SARS-CoV-2 neutralizing antibodies and its primary readouts: 50\% inhibitory concentration (IC50) by titration or single dilution screening at a sample dilution of 1:50. (A) Detection of inhibitors of the ACE2/SARS-CoV-2 spike interaction is achieved using an in-house purified and biotinylated human ACE-2 detector reagent. The ACE-2 binding signal is amplified by streptavidin-beta-galactosidase and a

566 fluorescent RGB-Substrate. (B) The entire assay is automated and performed using single molecule array 567 (SIMOA) technology on the Quanterix HD-X platform with a readout of average enzymes per bead (AEB). 


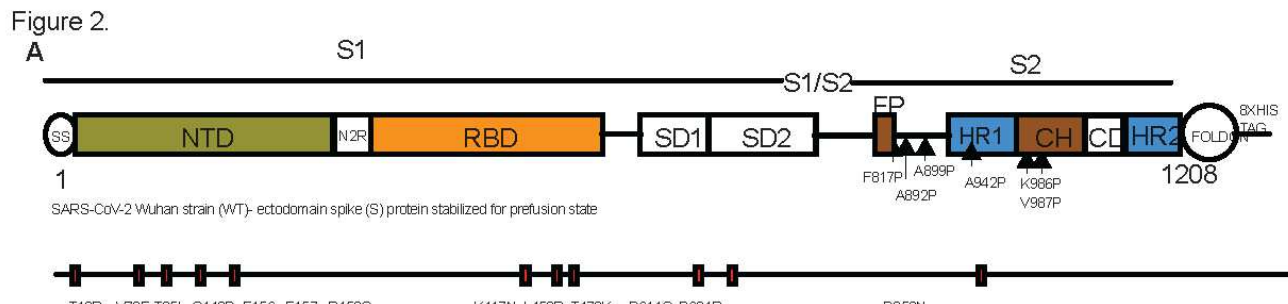

T19R, V70F, T951, G142D, E156, F157-,R158G, K417N, L452R, T478K, D614G, P681R, SARS-CoV-2B.1.617.2 (Detta strain)- ectodomain spike (S) protein stabllzed for prefusion stateDetta strain

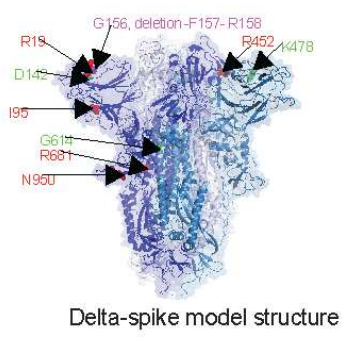

Delta-spike model structure
B

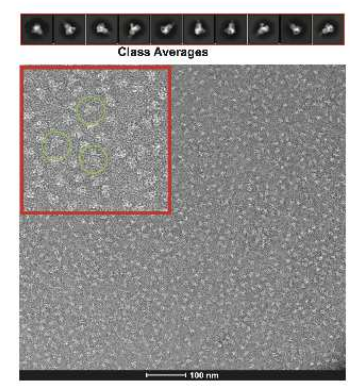

WT (Vaccine Strain)-Spike

C

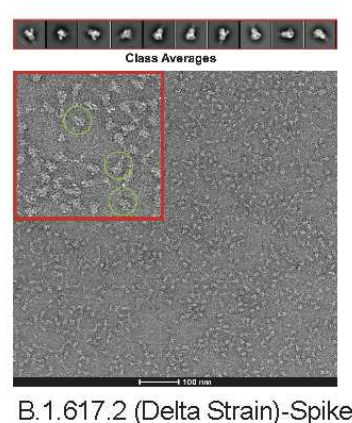

D SIDE

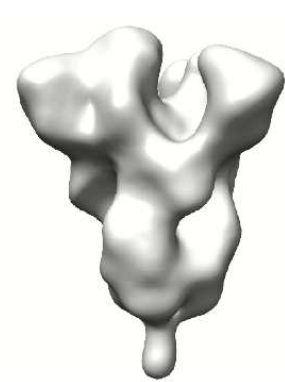

WT (Vaccine Strain)-Spike

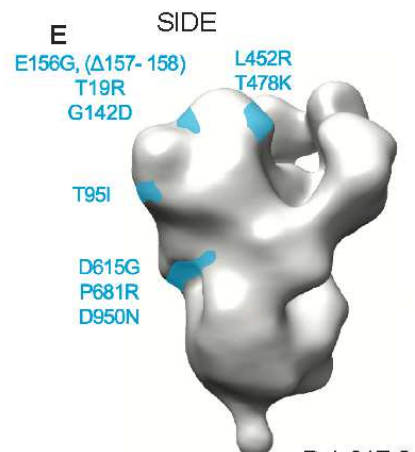

B.1.617.2 (Delta Strain)-Spike

APEX
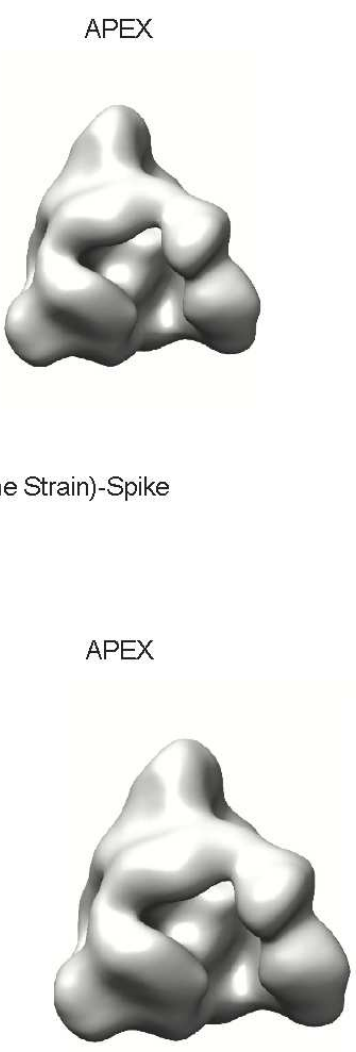

G BLI

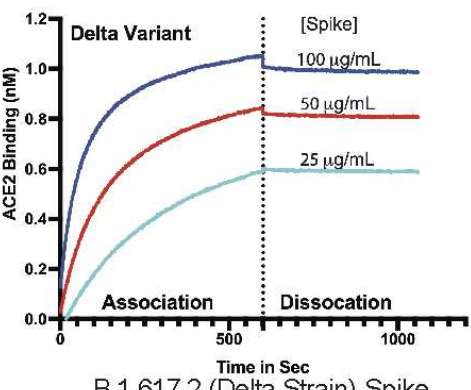

577 Figure 2. Development and validation of SARS-CoV-2 vaccine strain and delta variant prefusion

578 ectodomain spike targets for use in blockade of ACE-2 binding assay (BoAb). (A) SARS-CoV-2 spike (S)

579 ectodomain proteins of WT (Wuhan strain) and B.1.167.2 (Delta strain) for structural characterization and

580 assay development, and a model construct of Delta S protein. (B and C) Raw negative stain electron

581 micrographs for purified vaccine strain (B) and delta variant (C) trimers. Examples of native-like structures 
582 are encircled in the zoomed view, highlighted in a red cutout for each micrograph. 2D class averages of 583 various trimer orientations derived from the raw micrographs are shown in the upper panel and used for the

$5843 \mathrm{D}$ reconstruction shown in Panels C and D. (D) 3D reconstruction from negative stain electron microscopy

585 class averages of our purified vaccine strain trimeric ectodomain. (E) 3D reconstruction from negative stain

586 electron microscopy class averages of our purified delta variant (B.1.617.2) trimeric ectodomain with

587 significant amino acid substitutions mapped to the side view in light blue. (F,G) Biolayer interferometry

588 analysis of each spike variant binding to an immobilized recombinant ACE-2 IgG Fc-chimera, the

589 biotinylated form of which serves as the detector in our BoAb assay.

590

591

592

593

594

595

596

597

598

599

600

601

602

603

604

605

606

607 
A

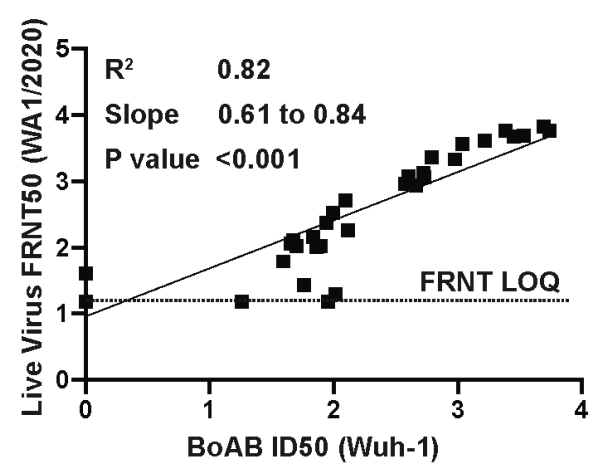

D

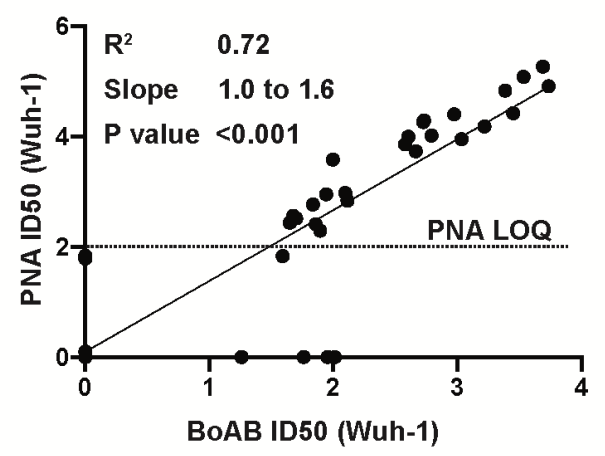

G

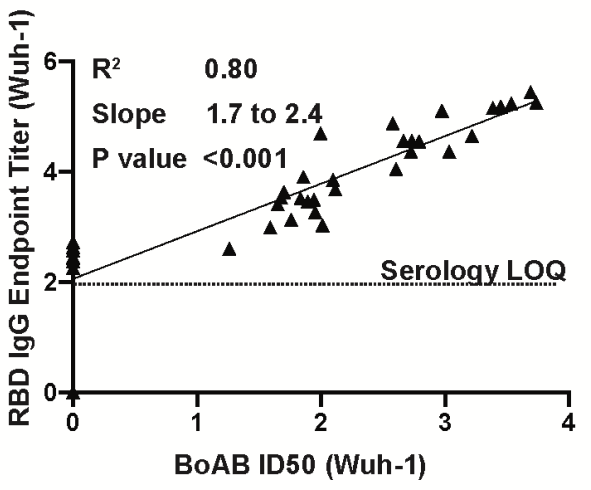

B

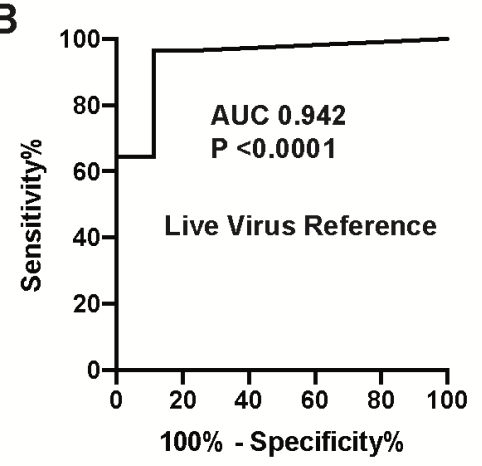

E

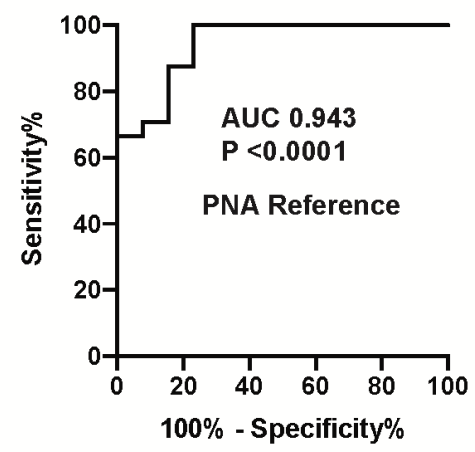

H

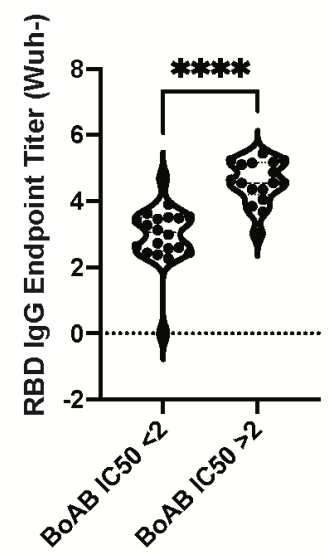

C

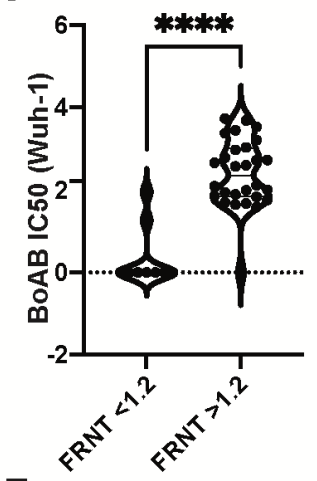

F

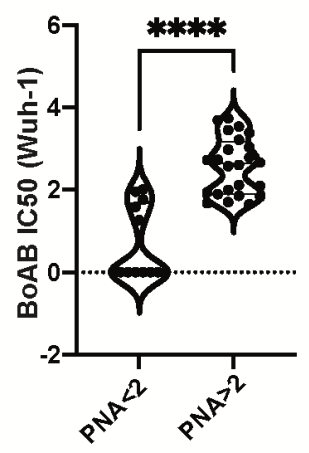

Figure 3. Correlation of vaccine strain BoAb IC50s with live virus and pseudovirus neutralization assays

611 (LVN and PNA). (A) Linear regression analysis of vaccine lineage (Wuh-1 \& WA1/2020) live virus 50\%

612 focus reduction neutralization activity (FRNT50) against ID50s in the vaccine strain BoAb. The absolute

613 value of the log dilution factor at which a sigmoidal curve (fit to duplicate eight-point dilution series for

614 each sample) crossed 50\% is plotted for the BoAb assay. (B,C) Receiver operator characteristic (ROC) 
615 curve and categorical comparison of the vaccine strain BoAb using a live virus FRNT cutoff of 1.17

616 (representing a linear dilution of 1 in 15) as the reference standard for neutralizing activity. (D) Linear

617 regression analysis of vaccine strain (Wuh-1 \& WA1/2020) pseudovirus 50\% inhibitory dilution (ID50)

618 against ID50s in the vaccine strain BoAb. (E,F) Receiver operator characteristic (ROC) curve and

619 categorical comparison of the vaccine strain BoAb using a pseudovirus neutralization ID50 of 2

620 (representing a linear dilution of 1 in 100) as the reference standard for neutralizing activity. (G) Linear

621 regression analysis of vaccine strain (Wuh-1 \& WA1/2020) receptor binding domain (RBD) specific IgG

622 titers against IC50s in the vaccine strain BoAb. Values are plotted as in (A) using an optical density cutoff

623 of 0.2 to quantify levels of binding antibodies. (H) Comparison of RBD IgG endpoint titers in samples with

624 vaccine strain BoAb activity less than or greater than a $\log$ IC50 of 2. Statistical significance was evaluated

625 by unpaired non-parametric $\mathrm{t}$ tests. $\mathrm{ns}=$ not significant, ${ }^{*} \mathrm{P}<0.05 ; * * \mathrm{P}<0.01 ; * * * \mathrm{P}<0.001 ; * * * * \mathrm{P}<0.0001$. 
A

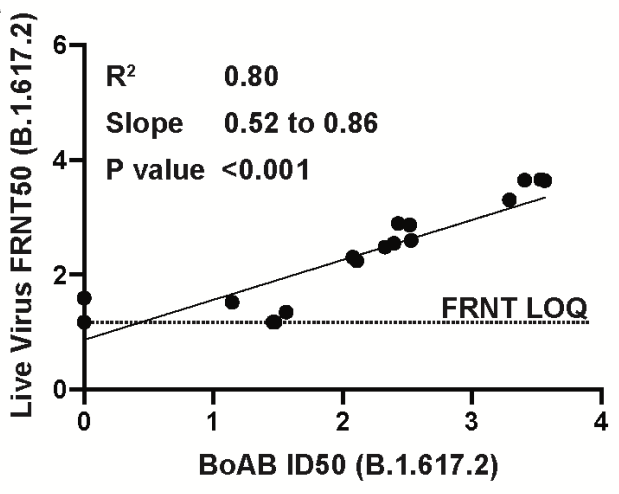

D

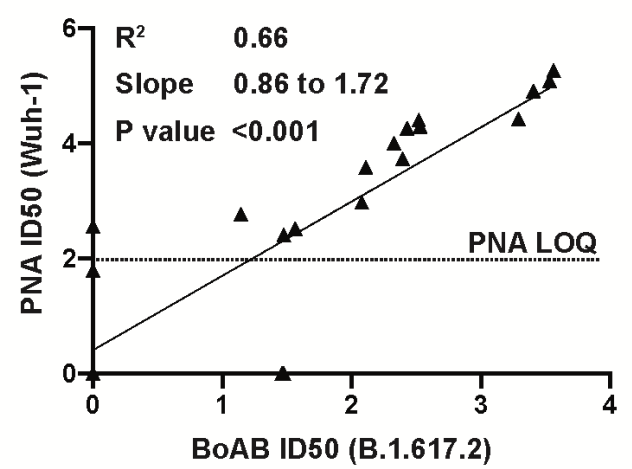

G

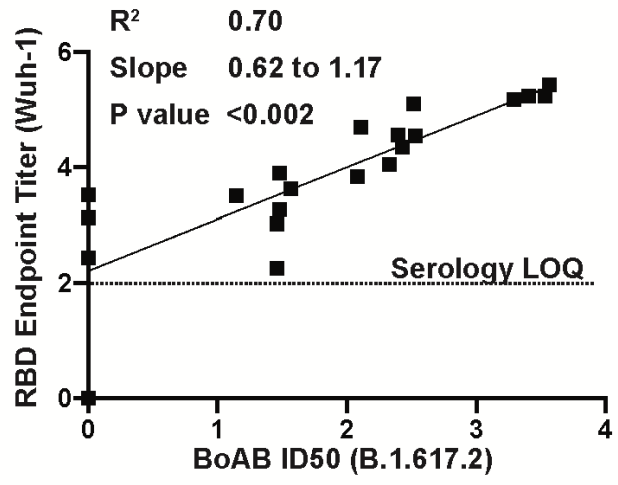

B

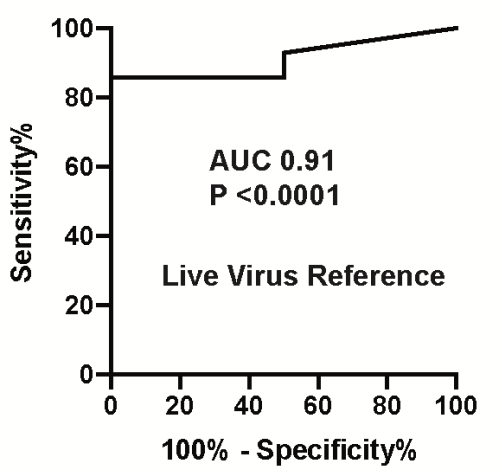

E

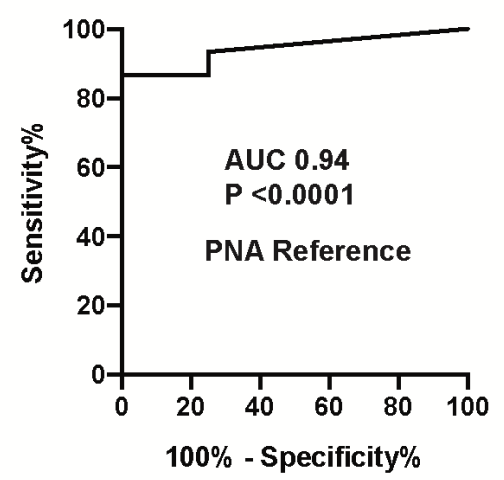

H

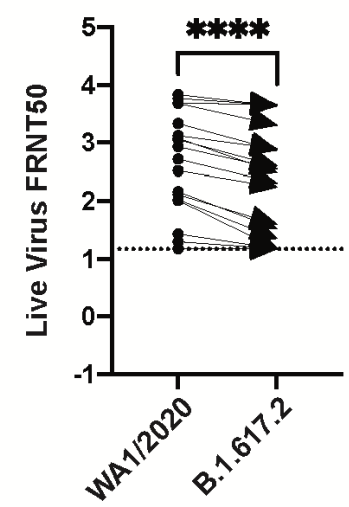

C

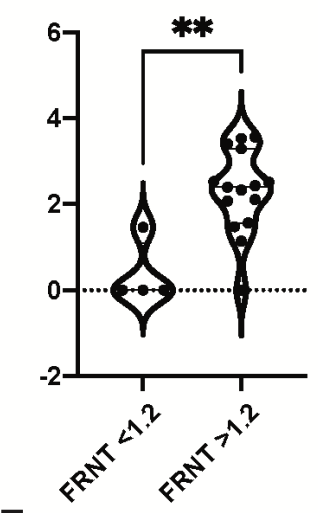

F

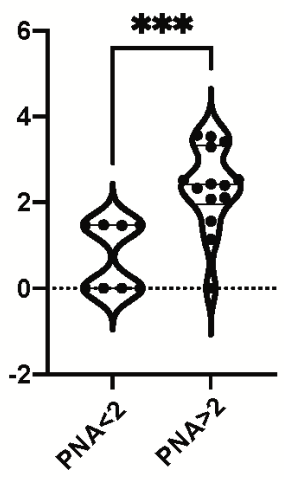

I

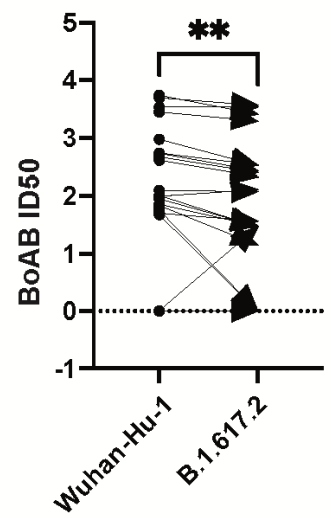

Figure 4. Correlation of delta variant BoAb ID50s with live virus and pseudovirus neutralization assays

644 (LVN and PNA). A) Linear regression analysis of delta variant (B.1.617.2) live-virus FRNT 50\% inhibitory

645 concentration (ID50) against ID50s in the delta variant BoAb. (B,C) Receiver operator characteristic (ROC)

646 curve and categorical comparison of the delta variant BoAb using a live virus FRNT cutoff of 1.17 
647 (representing a linear dilution of 1 in 15) as the reference standard for neutralizing activity. (D) Linear 648 regression analysis of vaccine strain (Wuh-1 \& WA1/2020) pseudovirus 50\% inhibitory concentration 649 (ID50) against ID50s in delta variant (B.1.617.2) BoAb. (E,F) Receiver operator characteristic (ROC) curve 650 and categorical comparison of the delta variant BoAb using a pseudovirus neutralization ID50 of 2 651 (representing a linear dilution of 1 in 100) as the reference standard for neutralizing activity. (G) Linear 652 regression analysis of vaccine strain (Wuh-1) receptor binding domain (RBD) specific IgG titers against 653 ID50 values in the delta variant BoAb. Values are plotted as in (A). (H,I) Paired comparison of live virus

654 FRNT50 and BoAb ID50 values between vaccine strain lineage (Wuhan-Hu-1 or WA1/2020) and delta 655 variant assays. Statistical significance was evaluated by paired non-parametric $t$ tests ns=not significant, 656 $* \mathrm{P}<0.05, * * \mathrm{P}<0.01, * * * \mathrm{P}<0.001, * * * * \mathrm{P}<0.0001$

A

B
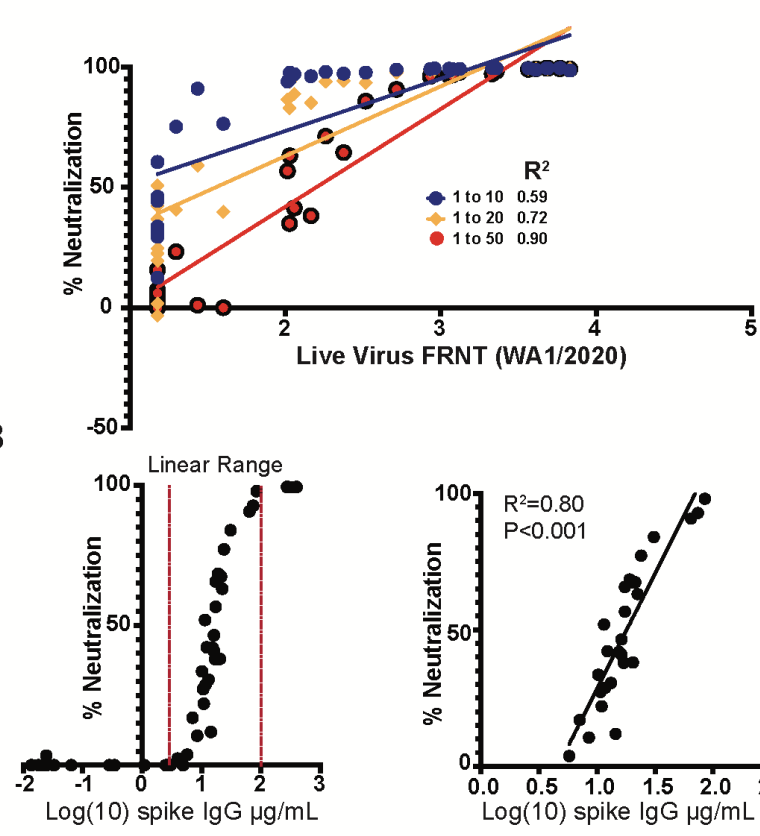
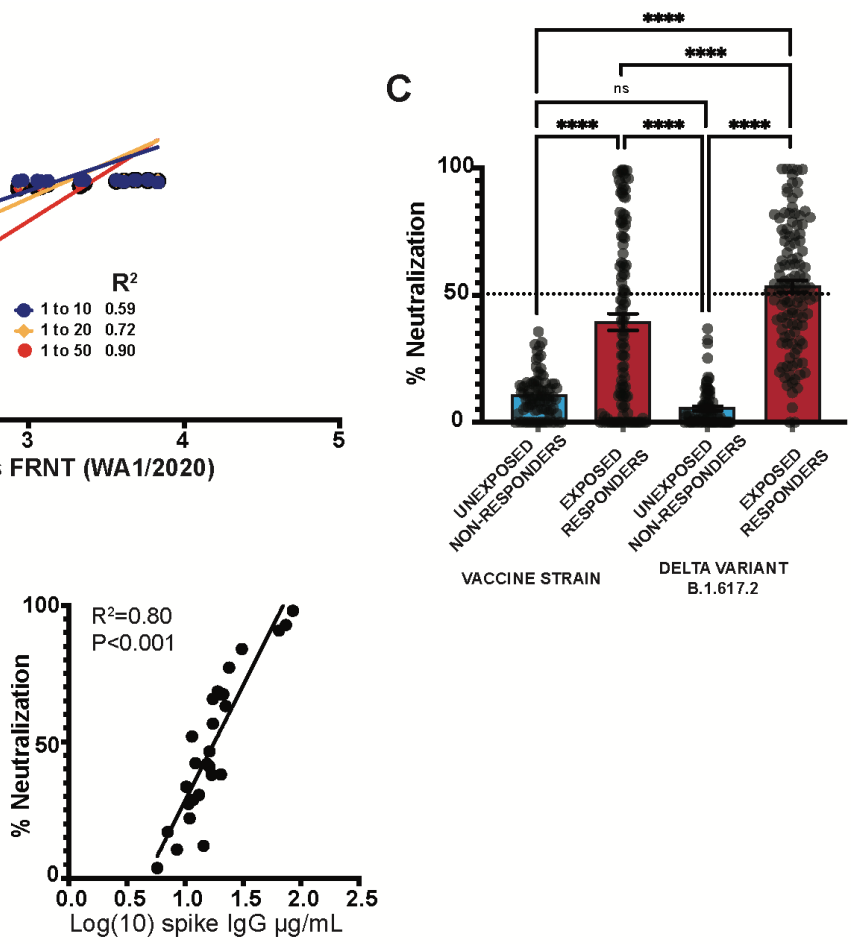

660 Figure 5. Detection of neutralizing antibodies by single dilution BoAb among SARS-CoV-2 exposed 661 patients (A) Screening by linear regression analysis of various single plasma dilution activities (1 to 10, 1 
662 to 20 , and 1 to 50) for correlation with WA1/2020 live virus FRNT ID50. R squared values are shown for

663 each regression. (B) Correlation of quantitative anti-spike IgG levels with 1:50 single dilution BoAb in all

664 tested vaccinated individuals. Additionally, the correlation was plotted with a separate linear regression that

665 was limited to samples with inhibition in the linear range $(C)$. Comparison of single dilution delta and

666 vaccine strain neutralizing antibody activity in patients with or without evidence of SARS-CoV-2 exposure

667 and seroconversion from a serosurvey conducted in the spring of 2021 at Emory University Hospital

668 Midtown. Statistical significance was evaluated by unpaired non-parametric $\mathrm{t}$ tests ns=not significant,

$669 * \mathrm{P}<0.05, * * \mathrm{P}<0.01, * * * \mathrm{P}<0.001, * * * * \mathrm{P}<0.0001$.

670

\section{Supplementary Figure 1.}

\section{RBD. ACE-2. MW (kDa). WT. Delta}

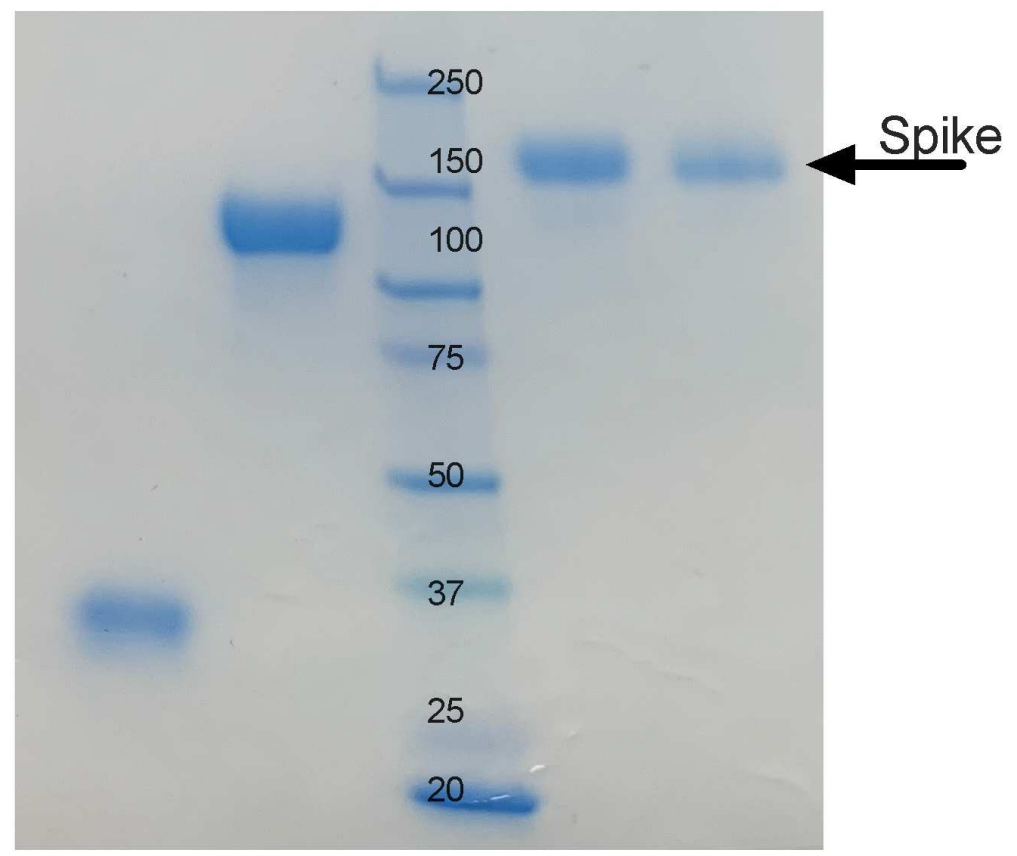

672 Supplementary Figure 1. SDS-PAGE for proteins from SARS-CoV-2 RBD, WT, Delta and hACE-2

673 expressed in Expi293 cells and purified by affinity and size exclusion chromatography. 